\title{
Intravenous infusion of small umbilical cord mesenchymal stem cells could enhance safety and delay retinal degeneration in RCS rats
}

Qingling Liang ${ }^{1,2^{*}}$, Qiyou Li ${ }^{1,2}$, Bangqi Ren ${ }^{1,2}$ and Zheng Qin Yin ${ }^{1,2^{*}}$

\begin{abstract}
Background: Human umbilical cord mesenchymal stem cells (UCMSCS) transplantation is a promising therapy for the treatment of retinitis pigmentosa (RP). However, intravenously infused cells may be blocked in the lung, increasing the risk of vascular obstruction, which needs to be optimized to further improve safety and efficacy.

Methods: We derived small UCMSCs (S-UCMSCs) from filtering UCMSCs with a 10- $\mu \mathrm{m}$ filter, and compared with UCMSCs by flow cytometry, directional differentiation culture and transcriptome sequencing. Then the S-UCMSCs and UCMSCs were intravenously infused in the Royal College Surgeons (RCS) rats to evaluate the safety and the efficacy.

Results: The diameter of S-UCMSCs ranged from 5.568 to $17.231 \mu \mathrm{m}$, with an average diameter of $8.636 \pm 2.256 \mu \mathrm{m}$, which was significantly smaller than that of UCMSCs. Flow cytometry, immunofluorescence and transcriptome sequencing demonstrated that the S-UCMSCs and UCMSCs were the same kind of MSCs, and the S-UCMSCs were more proliferative. After the S-UCMSCs and UCMSCs were intravenously infused into the Royal College of Surgeons $(\mathrm{RCS})$ rats at a dose of $1 \times 10^{6} \mathrm{cell} / \mathrm{rat}$, the S-UCMSCs blocked in the lungs were significantly fewer and disappeared more quickly than UCMSCs. The $b$ wave of the flash electroretinogram was improved at $7 d$, and the retinal outer nuclear layer thickness was thicker at $7 \mathrm{~d}$ and $14 \mathrm{~d}$. The expression level of inflammation was inhibited, and the expression level of neurotrophic factors was upregulated in the retina and serum after transplantation.
\end{abstract}

Conclusions: S-UCMSCs intravenous infusion was safer than UCMSCS and could delay retinal degeneration and protect visual function in RCS rats, which may be a preferable therapeutic approach for RP.

Keywords: Umbilical cord mesenchymal stem cells, Intravenous infusion, Safety, Efficacy, Retinitis pigmentosa

\section{Background}

Retinitis pigmentosa (RP) is a common form of hereditary retinal degenerative disease characterized by photoreceptor and retinal pigmented epithelium (RPE) degeneration [1, 2]. A variety of treatments, such as neurotrophic factors, visual prosthesis devices and gene therapy [3-5], are trying to treat RP. Recently, the first US Food and Drug Administration (FDA)-approved gene

*Correspondence: 846690411@qq.com; yzhengqin@163.com

2 Key Lab of Visual Damage and Regeneration \& Restoration, Chongqing 400038, China

Full list of author information is available at the end of the article therapy product, voretigene neparvovec-rzyl (Luxturna), was applied to the treatment of RPE65 gene-associated inherited retinal diseases, and promising results [6-8] have been encouraging for the treatment of RP.

Mesenchymal stem cells (MSCs) are multipotent stem cells characterized by self-renewal, multiple differentiation potentials, immunoregulation and neurotropic effects [9-11]. We have previously shown that bone marrow stem cells (BMSCs) could be induced toward functional retinal pigmented epithelium (RPE) cells or retinal neurons and secrete trophic factors under specific conditions in vitro [12, 13]. Therefore, MSC transplantation is considered to be a promising therapy for retinal original author(s) and the source, provide a link to the Creative Commons licence, and indicate if changes were made. The images or other third party material in this article are included in the article's Creative Commons licence, unless indicated otherwise in a credit line to the material. If material is not included in the article's Creative Commons licence and your intended use is not permitted by statutory regulation or exceeds the permitted use, you will need to obtain permission directly from the copyright holder. To view a copy of this licence, visit http://creativecommons.org/licenses/by/4.0/. The Creative Commons Public Domain Dedication waiver (http://creativeco mmons.org/publicdomain/zero/1.0/) applies to the data made available in this article, unless otherwise stated in a credit line to the data. 
degenerative diseases, such as wet age-related macular degeneration, Stargardt disease, and diabetic retinopathy $[14,15]$.

Intravenous infusion is a popular route for MSC transplantation in clinical trials $[16,17]$. For retinal disease, it is noninvasive, low-cost, convenient and avoids the probable complications caused by vitreous cavity and subretinal transplantation, such as proliferation of fibrous tissue and traction retinal detachment [18-20]. And because of these advantages, intravenous infusion can be conducted repeatedly according to the patients' condition, and it is more easily accepted and promoted by the public. Our previous clinical trials reported that intravenous infusion of MSCs could maintain or partially improve the visual function of advanced RP patients and nonproliferative diabetic retinopathy patients, and no side effects or complications were observed during these treatments [15, 21]. Furthermore, as RP is a kind of chronic progressive disease, along with the natural course of RP, the retinal microenvironment of patients with advanced RP is seriously damaged severely damaged, appearing ischemia and hypoxia, it is hardly to provide sufficient nutritional support or provide a good living environment for the intraocular transplanted cells. Therefore, intraocular transplantation may not be the most suitable treatment for advanced RP.

Umbilical cord mesenchymal stem cells (UCMSCs) are allogeneic MSCs derived from Wharton's jelly with low immunogenicity and more biological advantages [22], and they can be obtained more safely and conveniently than BMSCs obtained from donor bone marrow that may carry pathogenic genes. It is reported that UCMSCs possess immunomodulatory properties including impairing the phagocytic and antigen-presenting capacity of monocytes/macrophages, inhibiting the maturation of dendritic cells and the generation properties of CD4+ T helper (Th) 1 and Th17 cells, promoting the proliferation of Tregs and inhibiting the differentiation and antibody secretion of B cells [23-25]. And because of the immunosuppressive properties of UCMSCs, they have been used for the research of treatment of inflammatory disorders such as acute graft-versus-host disease, graft rejection in patients undergoing organ/cell transplantation, and autoimmune diseases etc., and no immune rejection was observed [26-28]. In addition, intravenous transplantation of UCMSCs can avoid immune rejection caused by retinal transplantation, so it may be the better choice for the treatment of RP.

However, some research has reported that the majority of intravascular infused MSCs might be blocked in the lungs and cause pulmonary vascular obstruction $[29,30]$, which increases the risk of transplantation. Although we ensured the safety of all subjects in our previous studies, with the increase in the number of subjects and number of administrations, there is also an increase in underlying risk of intravenous infusion of MSCs.

Therefore, further confirming the safety of intravenous infusion of UCMSCs and simultaneously maintaining their therapeutic role is our primary concern. In this study, we aimed to explore whether the intravenous infusion of small UCMSCs (S-UCMSCs) into Royal College of Surgeons (RCS) rats, a retinal degeneration animal model, could reduce the number of blocked cells in the lungs and simultaneously protect visual function.

\section{Methods}

\section{Animal model}

The Royal College of Surgeons (RCS) rat is the most classic and widely used animal model for studies of retinal degenerative disease [31, 32]. All RCS rats in this study are autosomal recessive rats with homozygous mutation of the tyrosine kinase Mertk gene, and they were provided by the Experimental Animal Center of the Army Medical University regardless of sex and raised in the Animal Care Center with free access to water and food. All animal experimental procedures were approved by the Institutional Review Board and the ethics committee of Southwest Hospital, Army Military Medical University, and conformed to the NIH guidelines for the ethical use of animals. All experiments involving human cells were carried out in accordance with the tenets of the Declaration of Helsinki, adhered to US Public Law 10341, effective December 13, 2001.

\section{Grouping of UCMSCs}

Human UCMSCs at passage 2 were obtained from Cyagen Biosciences, Suzhou, China, and all the cells met the following criteria. (1) Safety test criteria: asepsis, no endotoxin, no mycoplasma, and no hepatitis B or C; (2) quality test criteria: the positivity rate of CD11b, CD34 and CD45 was less than $5 \%$, and the positivity rate of CD44, CD73, CD90 and CD105 was greater than $80 \%$. The cell viability was not less than $90 \%$ [15]. A green fluorescence protein (GFP) lentiviral vector was used to transfect UCMSCs to track their distribution after intravenous infusion (Fig. S1). To obtain small UCMSCs, the cell suspension was filtered with a 10- $\mu \mathrm{m}$ filter (Sysmex, Japan), and the cells that passed through the pores were referred to as S-UCMSCs. The size of cells was evaluated by calculating the average diameter of 100 cells suspended in a culture dish by ImageJ software.

\section{Identification of MSC surface markers}

Flow cytometry was used to examine surface markers. UCMSCs and S-UCMSCs were suspended in staining buffer, and PE-labeled antibodies against CD73, D90 and 
CD105 and FITC-labeled antibodies against CD34, CD45 and HLA-DR were added. After incubation in the dark for $30 \mathrm{~min}$ and washing with phosphate-buffered saline (PBS), cells were analyzed on a fluorescence-activated cell sorting (FACS) instrument and analyzed with FlowJo software.

\section{Differentiation ability of cells}

UCMSCs and S-UCMSCs were cultured under specific conditions according to the manufacturer's instructions (Cyagen, China) to induce differentiation to osteocytes, chondrocytes and adipocytes at $37^{\circ} \mathrm{C}$ and $5 \% \mathrm{CO}_{2}$ for 30 days. Then, the differentiation potential was evaluated by staining with oil red $\mathrm{O}$ for adipogenesis, Alizarin red for osteogenesis and Alcian blue for chondrogenesis.

\section{Cell cycle detection}

Cells were prepared at a density of $1 \times 10^{6}$ cells/tube. After fixation in $70 \%$ ice-cold ethanol at $-20^{\circ} \mathrm{C}$ for $1 \mathrm{~h}$, $20 \mu \mathrm{l}$ RNase A solution was added and incubated for $30 \mathrm{~min}$ at $37^{\circ} \mathrm{C}$. Then, $400 \mu \mathrm{l}$ propidium iodide dyeing solution was added and incubated for $1 \mathrm{~h}$ at $4{ }^{\circ} \mathrm{C}$. FACS was performed at an excitation wavelength of $488 \mathrm{~nm}$ within $24 \mathrm{~h}$ after staining, and data were analyzed with ModFit software.

\section{Transcriptome sequencing}

Total RNA of S-UCMSCs and UCMSCs was extracted with TRIzol. cDNA libraries were sequenced on an Illumina sequencing platform by Genedenovo Biotechnology Co., Ltd. (Guangzhou, China). We analyzed the correlation between S-UCMSCs and UCMSCs based on the gene expression profile.

\section{Intravenous administration}

S-UCMSCs and UCMSCs were suspended at a density of $2 \times 10^{6}$ cells $/ \mathrm{ml}$ in $0.5 \mathrm{ml}$ balanced salt solution (BSS) and administered via the tail vein using a $30 \mathrm{G}$ needle to RCS rats (30 days old). RCS rats that received PBS or untreated rats served as the control groups. Three RCS rats in both the S-UCMSC group and UCMSC group were observed to evaluate the cells blocked in the lungs at 1, 12, 24, 48 and $72 \mathrm{~h}$ after transplantation, and 4 RCS rats in each experimental group were observed until 7, 14 and $28 \mathrm{~d}$ after transplantation to explore the efficacy of this treatment.

\section{Euthanize animals}

According to the experimental progress, at each observation time, the RCS rats of corresponding groups were placed in the carbon dioxide $\left(\mathrm{CO}_{2}\right)$ chamber and euthanized within $5 \mathrm{~min}$ as the $\mathrm{CO}_{2}$ filled the chamber at a rate of 10 to $30 \%$ of the box volume per minute.

\section{Counting GFP-positive cells in the lung}

The whole lung was immediately removed when RCS rats were sacrificed at $1,12,24,48$ and $72 \mathrm{~h}$ after transplantation to make frozen sections, and DAPI staining was performed. To analyze the number of cells blocked in the lung, in the 5 lobes, 3 high power fields $(\times 400)$ were randomly selected from every lobe of each lung and observed by confocal laser scanning microscopy. The number of nuclei covered with green fluorescence was statistically analyzed.

\section{Stretched preparation and frozen sectioning of the retina}

Eyes were obtained for retinal preparation at 1, 12, 24, 48 and $72 \mathrm{~h}$ after transplantation. After fixation in $4 \%$ PFA for $3 \mathrm{~h}$, the whole retina was removed and placed on glass slides. At 7, 14 and 28 days after transplantation, the eyes were sectioned at $10 \mu \mathrm{m}$ through the optic nerve along the vertical meridian within a radius of $1 \mathrm{~mm}$ to make frozen sections of the retina. To evaluate the degeneration of photoreceptors, we randomly selected 10 tissue samples and measured the outer nuclear layer $(\mathrm{ONL})$ thickness with ImageJ software.

\section{Electrophysiological examination}

Flash electroretinogram (FERG) examination was performed with a visual electrophysiology instrument (MAYO, Japan) before animals were sacrificed and at 7 , 14 and 28 days after transplantation. The amplitude and latent time of the b wave were recorded at an intensity of $3.0(\mathrm{P})$ d.s $/ \mathrm{m}^{2}$.

\section{ELISA assay}

When the RCS rats were sacrificed at 7, 14 and 28 days after transplantation, the RPE-Bruch's membrane choriocapillaris complexes were removed carefully and homogenized, and the supernatant was collected after centrifugation. Blood samples of the RCS rats were obtained from the left ventricle, and serum was collected after centrifugation.

After the samples were collected, ELISAs were performed according to the manufacturer's instructions (Cusabio, China) for interleukin-6 (IL-6), interleukin-10 (IL-10), brain-derived neurotrophic factor (BDNF), ciliary neurotrophic factor (CNTF), basic fibroblast growth factor (bFGF) and hepatocyte growth factor (HGF).

\section{Statistical analysis}

Descriptive statistics were performed using GraphPad Prism 6.0. Data are presented as the mean \pm SD. Student's t-test was used for two groups, while twoway ANOVA was used for multiple data analysis and 
statistical analysis. A value of $P<0.05$ was considered statistically significant.

\section{Results}

After intravenous infusion of S-UCMSCs and UCMSCs into RCS rats through the tail vein, no abnormal reactions in daily dietary activities were observed, and no immune rejection or tumorigenesis in the retina were found by stretch preparation or frozen section throughout the 28 days.

\section{Biological characteristics of S-UCMSCs and UCMSCs}

The size of cultured UCMSCs at passage 5 in suspension conditions ranged from 6.364 to $39.395 \mu \mathrm{m}$, with an average diameter of $16.205 \pm 5.947 \mu \mathrm{m}$ (Fig. 1a). The size of S-UCMSCs that passed through 10- $\mu$ m nylon filters ranged from 5.568 to $17.231 \mu \mathrm{m}$, with an average diameter of $8.636 \pm 2.256 \mu \mathrm{m}$, which was significantly smaller than that of UCMSCs (Fig. 1b). Surface antigen detection showed that UCMSCs and S-UCMSCs shared a similar surface antigen pattern: both were highly positive for CD73, D90 and CD105 and negative for CD34, CD45 and HLA-DR (Fig. 1c), which meets the minimal criteria for defining MSCs [33].

After 30 days of adipogenic differentiation, the morphology and quantity of oil red O-positive lipid vacuoles in UCMSCs were very similar to those in S-UCMSCs. Both kinds of cells could be induced to differentiate into cells with similar mineralized nodules (stained with Alizarin red) in osteogenic differentiation assays and with proteoglycan pellets (stained with Alcian blue) in chondrogenic differentiation assays (Fig. 1d).

The proliferative capacity of UCMSCs was assessed by the proliferation index $(\mathrm{PI}=(\mathrm{S}+\mathrm{G} 2 / \mathrm{M}) /$ $(\mathrm{G} 0 / 1+\mathrm{S}+\mathrm{G} 2 / \mathrm{M})$. The percentage of cells in each cell cycle phase revealed that more S-UCMSCs than UCMSCs were in S phase, but fewer were in G0/1 and G2/M phases (Fig. 1e and $\mathrm{f}$ ). The PI of S-UCMSCs was significantly higher than that of UCMSCs (Fig. 1g).

Transcriptome sequencing showed that UCMSCs and S-UCMSCs were closely related in the correlation heat map (Fig. 2a), and the Pearson correlation coefficient of the two groups of cells was 0.9976 (Fig. 2b), which demonstrated that S-UCMSCs and UCMSCs were the same kind of cells with similar gene expression profiles, despite the upregulation of 53 genes and downregulation of 40 genes (Fig. 2c).

\section{Fewer S-UCMSCs than UCMSCs were blocked in the lungs of $\mathrm{RCS}$ rats}

S-UCMSCs and UCMSCs were intravenously infused into RCS rats through the tail vein, and the lungs were collected at 1, 12, 24, 48 and $72 \mathrm{~h}$ after transplantation. As shown in Fig. 3, $1 \mathrm{~h}$ after transplantation, a large number of GFP-positive cells were blocked in the lungs in both groups. Then, the number of S-UCMSCs blocked in the lung quickly decreased (Fig. 3a and b), and significantly fewer S-UCMSCs than UCMSCs were in the lungs at $12 \mathrm{~h}$ and $24 \mathrm{~h}$ after transplantation (Fig. 3c). Forty-eight hours later, S-UCMSCs had almost disappeared, with too few cells retained in the lung for statistical analysis, while residual UCMSCs could still be observed (Fig. S2). Furthermore, no GPF-positive cells were observed in the lungs at $72 \mathrm{~h}$ after transplantation.

\section{S-UCMSCs and UCMSCs have similar protective effects in $\mathrm{RCS}$ rats}

Flash Electroretinogram (FERG) tests showed that the average amplitude/latency $(\mathrm{A} / \mathrm{L})$ ratio of the $\mathrm{b}$ wave of the UCMSC and S-UCMSC groups was higher than that of the PBS and untreated groups at 7 and 14days after intravenous infusion (Fig. 4a), and the difference at 7 days was statistically significant (Fig. $4 \mathrm{~b}$ ). With the progression of retinal degeneration, the amplitude of the $b$ wave was hardly observed at 28 days in all groups.

The ONL thickness of the S-UCMSC and UCMSC groups was significantly thicker than that of the PBS and untreated groups at 7 days and 14 days after transplantation (Fig. 4c and d). With the natural course of retinal degeneration, only approximately $3-5$ layers remained in the ONL in all groups 28 days after transplantation.

The mechanism of UCMSC intravenous transplantation is mainly attributed to anti-inflammatory and neurotrophic effects [34, 35]. The ELISA showed that the expression level of IL-6 in the RPE-Bruch's membrane choriocapillaris complexes of the RCS rats' retina in the S-UCMSC and UCMSC groups was significantly lower and the level of IL-10 was much higher

(See figure on next page.)

Fig. 1 Comparison of the morphology and biological characterization of S-UCMSCS and UCMSCs. a In suspension culture, S-UCMSCS were significantly smaller than UCMSCs. b Statistical analysis of the average diameter of S-UCMSCs and UCMSCs $(n=100)$. c Flow cytometry showed that surface antigens of both S-UCMSCs and UCMSCs were positive for CD73, D90 and CD105 and negative for CD34, CD45 and HLA-DR. d Both S-UCMSCS and UCMSCs could be induced to differentiate into adipocytes, osteocytes and chondrocytes, which was confirmed by oil red O, Alizarin red and Alcian blue staining. e Representative cell cycle histograms from FACS. $\mathbf{f}$ The proportion of cells in each cell cycle phase $(n=3)$. $\mathbf{g}$ Statistical analysis showed that the PI of S-UCMSCs was higher than that of UCMSCs $(n=3) .{ }^{*} P<0.05,{ }^{* * *} P<0.001$ versus UCMSCs. Scale bars: $20 \mu \mathrm{m}(a)$, $100 \mu \mathrm{m}$ (D1 and D2), $200 \mu \mathrm{m}$ (D3 and D4), and $50 \mu \mathrm{m}$ (D5 and D6) 


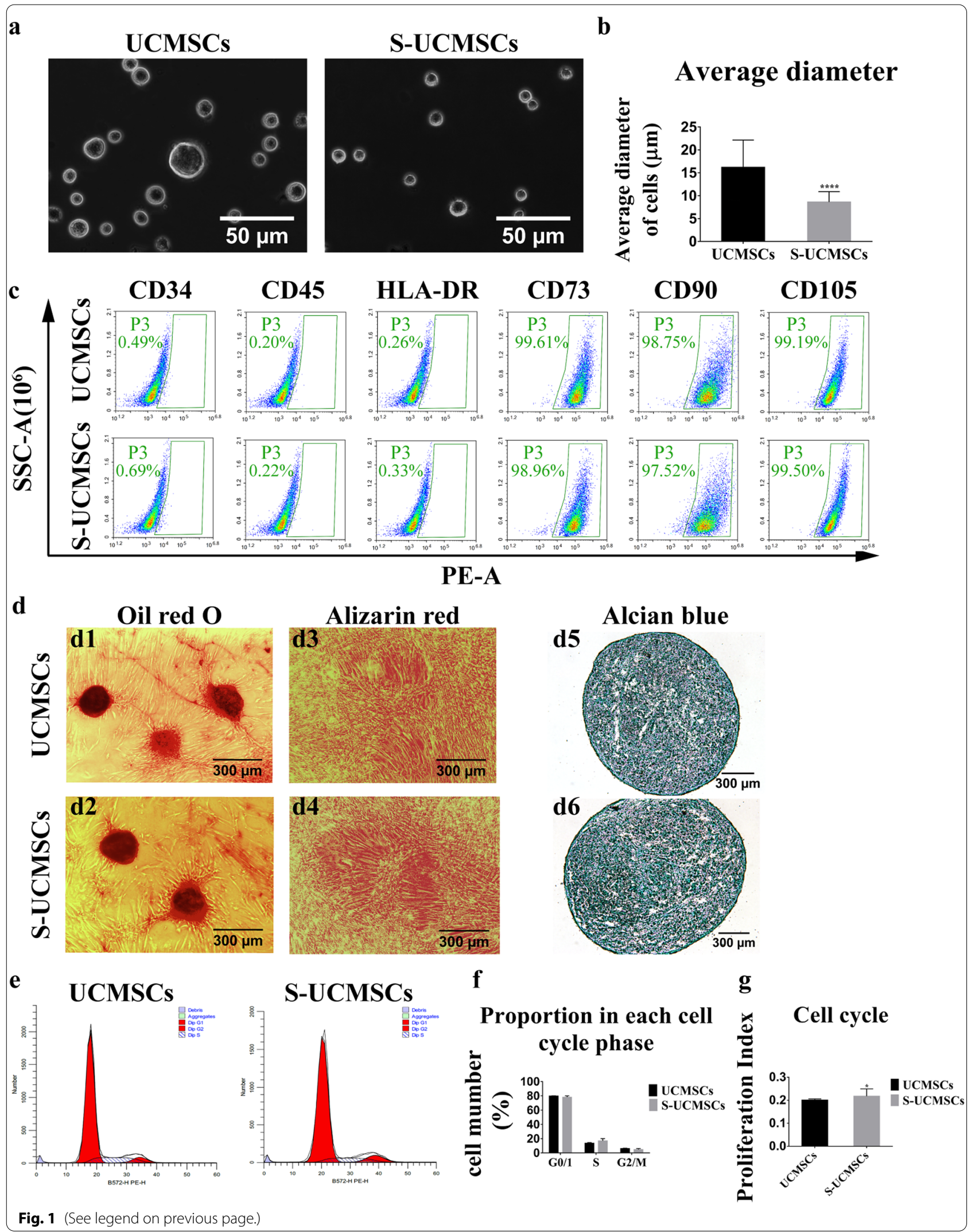




\section{a}

\section{Sample Correlation}
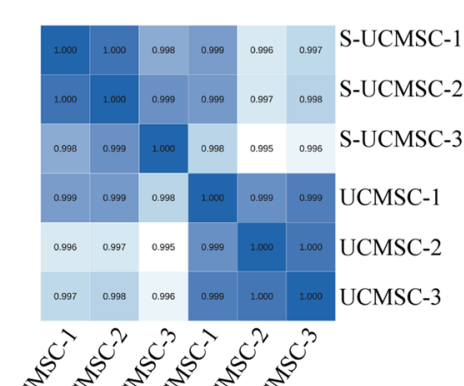

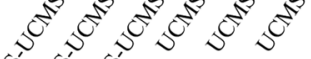

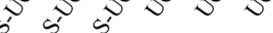

Fig. 2 Correlation analysis of gene expression by transcriptome sequencing. a The correlation heat map demonstrated that the gene expression profiles of S-UCMSCS and UCMSCs were very similar. b The scatter plot showed that the Pearson correlation coefficient was 0.9976 between UCMSCs and S-UCMSCS. c Statistical analysis of differentially expressed genes showed that 53 genes were upregulated and 40 genes were downregulated in UCMSCs versus S-UCMSCS

\section{b}

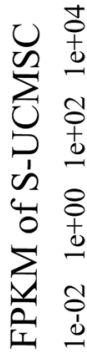

1e-02 $\quad 1 \mathrm{e}+00 \quad 1 \mathrm{e}+02 \quad 1 \mathrm{e}+04$
C

DiffExp Gene Statisitics

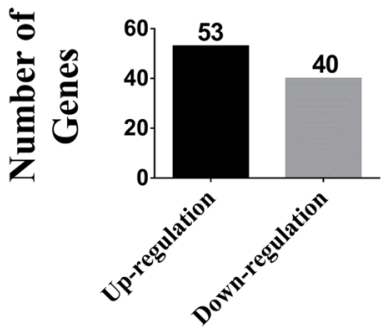

$\mathbf{a}$

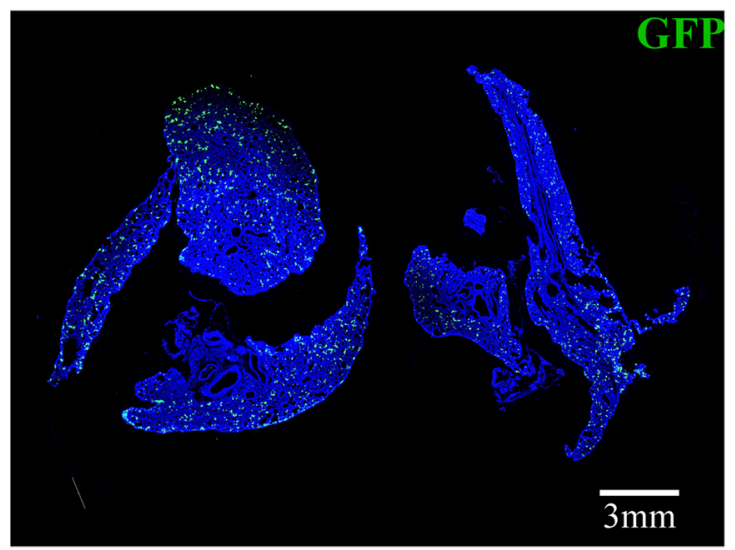

b

c

$12 \mathrm{~h}$

$24 \mathrm{~h}$

$48 \mathrm{~h}$
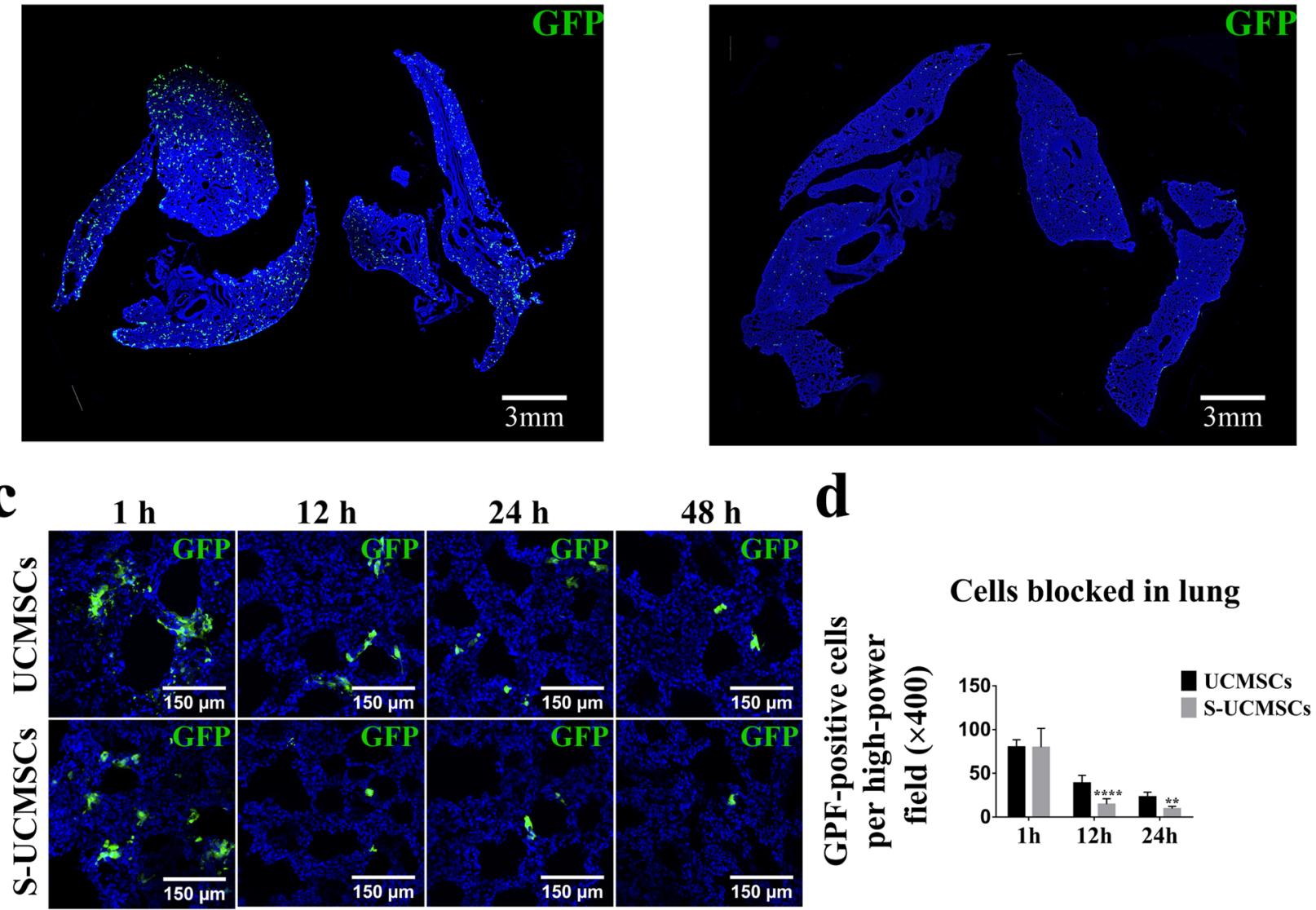

Fig. 3 Comparison of the number of blocked cells in the lungs after transplantation. Representative micrographs of the whole lung fields of the S-UCMSC group at $1 \mathrm{~h} \mathrm{(a)} \mathrm{and} 24 \mathrm{~h}(\mathbf{b})$ showed that the number of blocked S-UCMSCs in the lung decreased rapidly after transplantation. c There were many GFP-positive cells in both the S-UCMSC and UCMSC groups at $1 \mathrm{~h}$ after transplantation, and there were significantly fewer S-UCMSCS blocked in the lungs than UCMSCs at $12 \mathrm{~h}$ and $24 \mathrm{~h}$. $\mathbf{d}$ Statistical analysis of GFP-positive cells blocked in the lungs after transplantation $(\mathrm{n}=3)$. ${ }^{*} P<0.01 ;{ }^{* * *} P<0.001$ versus UCMSCs. Scale bar: $3 \mathrm{~mm}(\mathbf{a}$ and $\mathbf{b})$ and $100 \mu \mathrm{m}(\mathbf{c})$ 


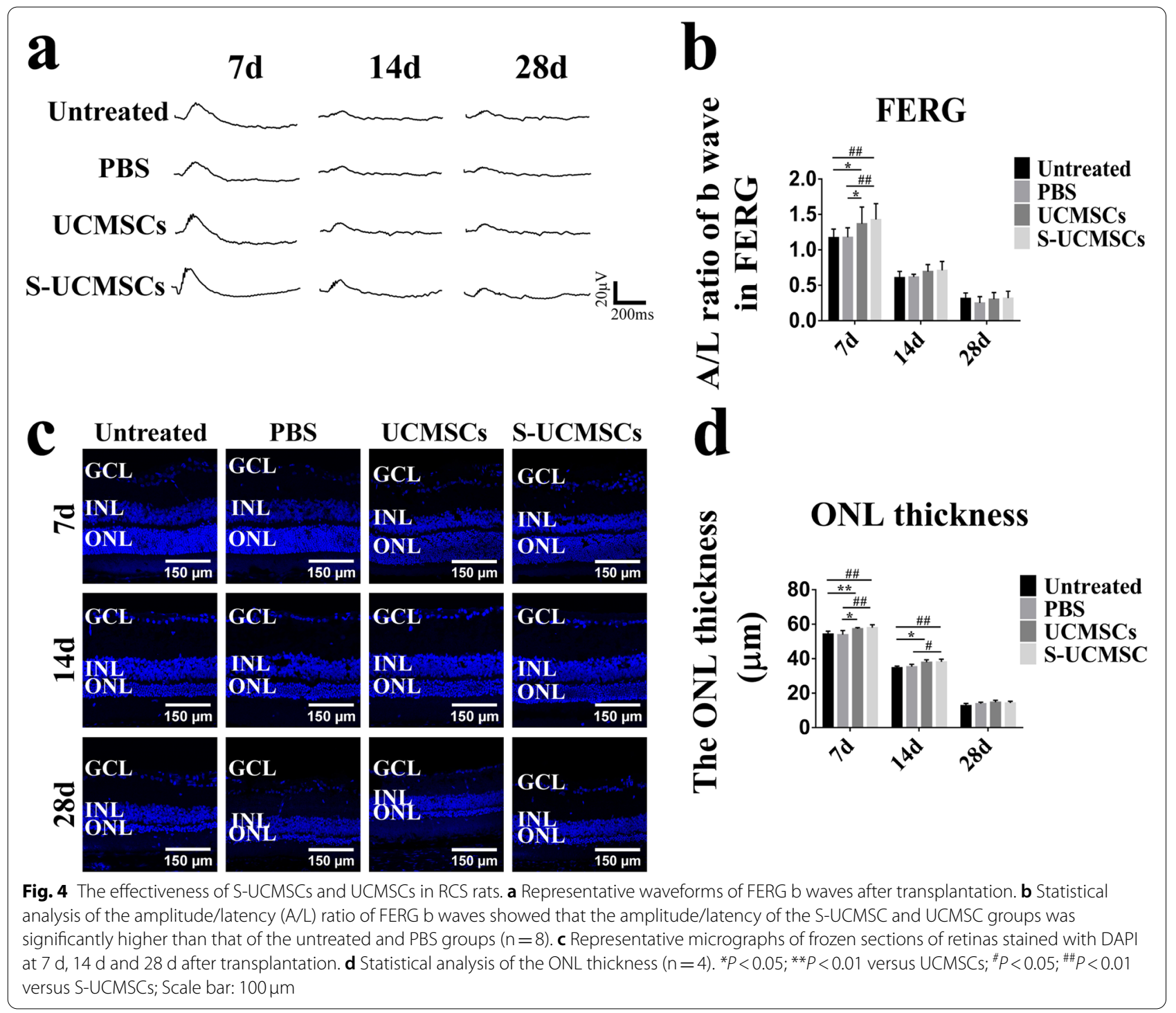

than those in the PBS and untreated groups after transplantation (Fig. 5a and b). Meanwhile, the levels of BDNF in the two transplanted groups were significantly higher at $14 \mathrm{~d}$ and that of bFGF was upregulated at $7 \mathrm{~d}$ and $14 \mathrm{~d}$ compared with the two control groups, and the levels of CTNF and HGF were significantly continuously upregulated until 28 days after transplantation (Fig. 5c-f).

Furthermore, we found that the level of IL- 6 in the serum of RCS rats in the S-UCMSC and UCMSC groups was lower than that in the PBS and untreated groups at 7 days, and the difference with the PBS group was significant (Fig. 6a). The level of IL-10 tended to increase at 7 and 14 days after transplantation (Fig. 6b). Meanwhile, the expression levels of BDNF, CNTF and bFGF in the two transplanted groups were significantly upregulated at $14 \mathrm{~d}$ or $28 \mathrm{~d}$ after treatment (Fig. 6c-f).
In addition, compared with UCMSCs, S-UCMSCs displayed no significant advantage in regard to the therapeutic effect.

\section{Discussion}

UCMSCs are an ideal cell source for stem cell transplantation [36]. Compared with MSCs derived from the bone marrow, adipose tissue and other sources, UCMSCs avoid ethical controversy, and they are derived through noninvasive means without pathogenic genes carried by the donor [37, 38]. Studies have reported that UCMSC transplantation can be safely applied to a variety of diseases and has shown some positive effects [39-42]. However, evidence has shown that the majority of intravenously delivered MSCs are blocked inside the lungs, which might cause pulmonary vascular obstructions to increase the risk of transplantation [43, 44]. Therefore, it is necessary 

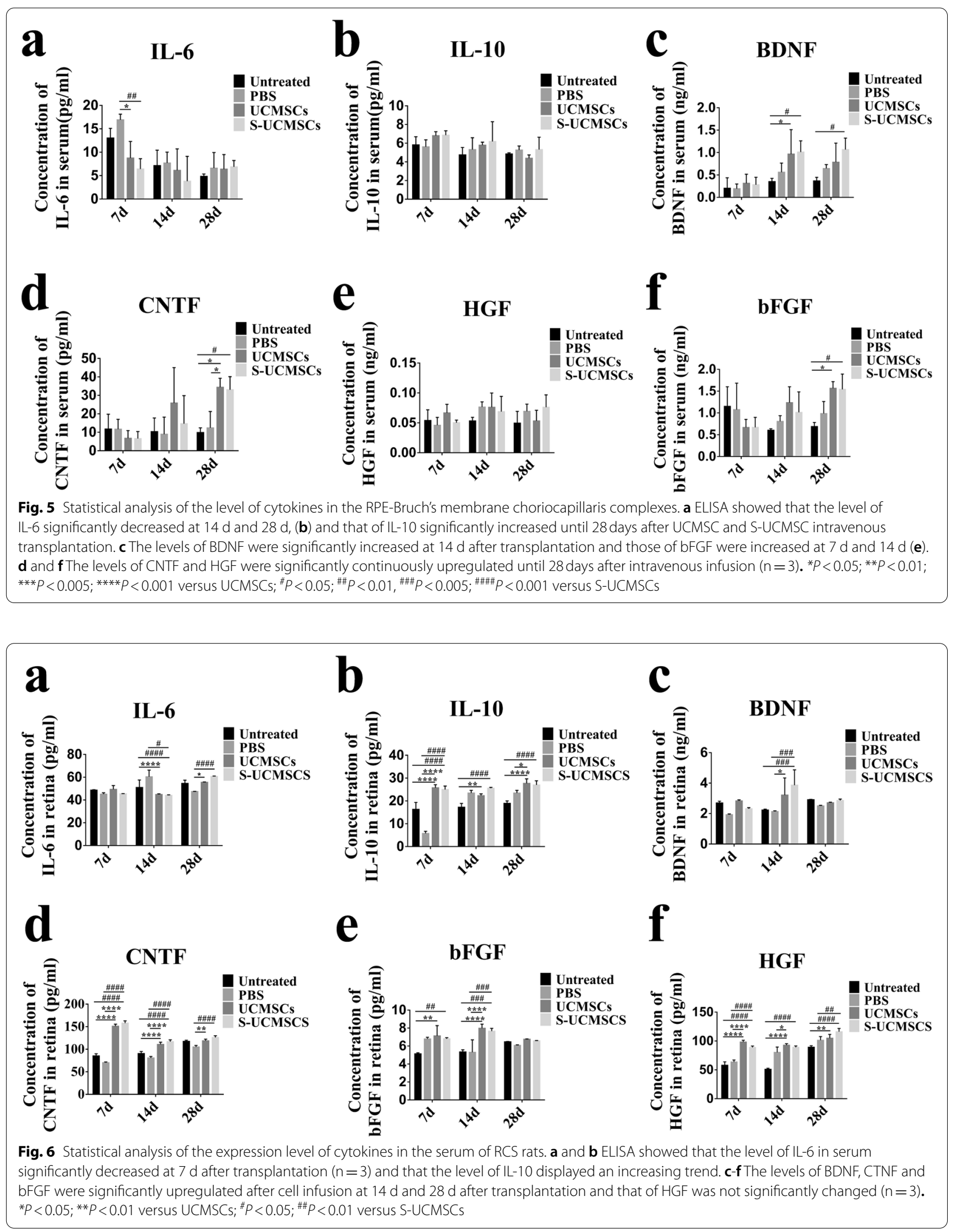
to optimize MSC intravenous infusion to further improve safety. Although the mechanisms of vascular obstruction are poorly understood, the size of cells has been assumed to be the key factor [45]. We obtained S-UCMSCs by filtering UCMSCs with a 10- $\mu \mathrm{m}$ nylon filter. Compared with UCMSCs, S-UCMSCs were more proliferative. Intravenously infused S-UCMSCs were less blocked in the lung and disappeared faster than UCMSCs. Moreover, S-UCMSCs provided retinal protection similar to that of UCMSCs.

To our knowledge, no studies have explored the safety and efficacy of the intravenous infusion of S-UCMSCs in RCS rats. We previously conducted phase II clinical trials on the intravenous infusion of MSCs in RP and diabetic retinopathy patients, and safety and promising therapeutic effects were observed. In addition, the safety of intravenous infusion has also been proven by meta-analysis [16] and other clinical studies [46, 47]. Therefore, intravenous infusion of UCMSCs is regarded as a safe approach based on the large number of previous studies and our clinical trial. However, no drugs or approaches can guarantee absolutely safe even if they have been widely applied to clinical treatment, which is the case for intravenous infusion. Unfortunately, as pulmonary capillaries with an average diameter of $6 \sim 9 \mu \mathrm{m}$ are too small for UCMSCs with an average diameter of $16.205 \pm 5.947 \mu \mathrm{m}$ to pass through, they are considered to be the major obstacle for intravascular infused cells to migrate to other tissues $[44,48]$. Therefore, to make the cells that infused intravenously pass through the pulmonary capillaries as many as possible, a $10 \mu \mathrm{m}$ nylon filter was selected, which is the closest available size to the capillary diameter to obtain the S-UCMSCs. In this study, we clearly found that significantly fewer S-UCMSCs, with an average diameter of $8.636 \pm 2.256 \mu \mathrm{m}$, were blocked in the lung than UCMSCs at $12 \mathrm{~h}$ and $24 \mathrm{~h}$ after transplantation, which might reduce the risk of vascular embolism. Consistent with our observations, Zanetti et al. [49] revealed that smaller BMSCs, with a diameter of $15.1 \pm 0.9 \mu \mathrm{m}$, had an increased capacity to pass through the pulmonary microcirculation compared with traditional MSCs, and Ge et al. [50] also pointed out that the size of intrainternal artery-injected MSCs is a significant cause of vascular obstructions and stroke. Therefore, small MSCs may be a key factor in improving the safety of intravenous transplantation. Moreover, similar to other experiments $[45,51]$, we observed that S-UCMSCs displayed a more active proliferative state, and this superiority might play an important role in their rapid passage through the lung and therapeutic function.

The effectiveness of MSCs for the treatment of retinal degeneration has been proven in our previous studies $[19,52]$. Then, did the difference in UCMSC size influence their function? In the present study, we observed that S-UCMSCs could protect visual function and photoreceptors by improving the FERG $b$ wave and reducing the loss of ONL cells, which indicated that S-UCMSCs could delay retinal degeneration as effectively as UCMSCs. However, interestingly, neither S-UCMSCs nor UCMSCs homing to the retina were observed after intravenous infusion (Fig. S3). This might largely be attributed to obstruction of the lungs, while the blood retinal barrier might also be one of the reasons. Indeed, the homing of MSCs has always been discussed. Some studies demonstrated that intravenously administered MSCs could migrate to the sites of injury mediated by chemokines and the recruitment of the injured tissue [53-55]. However, Eggenhofer et al. [29] reported that MSCs are short-lived and do not migrate beyond the lungs after intravenous infusion, which was similar to our findings. We observed that despite being short-lived (less than $72 \mathrm{~h}$ ), the efficacy of S-UCMSCs and UCMSCs, including maintaining visual function and protecting photoreceptors from degeneration, lasted approximately 28 days after transplantation, which was much longer than their presence. It is most likely that intravenous infusion of S-UCMSCs and UCMSCs can continuously regulate the retinal microenvironment through immunomodulatory and neurotrophic effects mediated by paracrine mechanisms, such as decreasing the level of IL-6 and upregulating the level of IL-10 and neurotrophic factors, such as BDNF, CNTF, bFGF and HGF, rather than homing to the retina and differentiating and replacing damaged tissues and cells $[1,56]$. However, the validity period of 28 days in this study is limited for the treatment of $\mathrm{RP}$, a chronic progressive disease. Although the differences of the species of objects and research methods are important factors for the results, and our previous clinical study of intravenous transplantation of UCMSCs in RP patients showed that the effectiveness lasted for 12 months, it is still necessary to extend the observation time in the future studies to further explore the duration of effectiveness.

However, the lack of research on the distribution of other tissues of intravenously infused cells in addition to the lung and retina might be a limitation of our study. Since the lung is the organ in which the cells are blocked most heavily compared with other organs and pulmonary complications are more likely to be life-threatening, we focused on the lung in the present study. Nonetheless, the fate and migration pathway of intravenously infused UCMSCs still need to be further explored in subsequent studies to better clarify the therapeutic mechanism and optimize the treatment. In addition, although no cells migrated to the retina in our study, in view of the considerable controversy about cell homing, multiple 
experimental methods are still needed to further confirm our research results.

In addition, although various approaches for small MSCs, such as filters, centrifuge system, counter flow elution and microfluidic sorting $[45,57,58]$, have been developed in addition to nylon filters, there is a lack of unified processes and standards for their preparation, which limits the application of small MSCs. Therefore, based on maintaining biological function, methods to standardize the process and unify cell size for clinical application need to be further studied.

\section{Conclusions}

In summary, compared with UCMSCs, intravenous infusion of S-UCMSCs was safer and could protect visual function and delay retinal degeneration in RCS rats. This study provides a novel strategy to optimize UCMSC transplantation for RP patients, especially when administered repeatedly and the range of subjects expands.

\section{Abbreviations}

S-UCMSCs: Small Umbilical Cord Mesenchymal Stem Cells; ONL: Outer Nuclear Layer; FERG: Flash Electroretinogram; BDNF: Brain-Derived Neurotrophic Bactor; CNTF: Ciliary Neurotrophic Factor; bFGF: Basic Fibroblast Growth Factor; HGF: Hepatocyte Growth Factor.

\section{Supplementary Information}

The online version contains supplementary material available at https://doi. org/10.1186/s12886-021-02171-3.

Additional file 1: Figure S1. The expression of GFP in UCMSCs. Figure S2. Distribution of GFP-positive cells in whole lung fields. Figure S3. Representative micrograph of retinal preparation.

\section{Acknowledgements}

The authors thank Yijian Li and LiNa Wang for assistance in animal experiments and Wei Li for assistance in figures editing.

\section{Authors' contributions}

LQL wrote the manuscript and prepare all the figures. $L Q L, L Q Y$ and RBQ performed the major examination and analyzed the data. YZQ designed the study and conducted the writing. All authors read and approved the final manuscript.

\section{Funding}

This work was supported by the Military Key Program (BWS13C015).

\section{Availability of data and materials}

The datasets used and/or analyzed during the current study are available from the corresponding author on reasonable request.

\section{Declarations}

\section{Ethics approval and consent to participate}

This study was carried out in accordance with the Declaration of Helsinki. All experiments and methods were approved and performed in accordance with relevant guidelines and regulations of the Ethics Committee of Army Medical University of China. All animal experimental procedures were approved by the Southwest Hospital committee for experiments in animals and adhered to the NIH guidelines for the ethical use of animal. This study was carried out in compliance with the ARRIVE guidelines.

\section{Consent for publication}

Not Applicable.

\section{Competing interests}

The authors declare that they have no competing interests.

\section{Author details}

${ }^{1}$ Southwest Hospital/Southwest Eye Hospital, Third Military Medical University (Amy Medical University), Chongqing 400038, China. ${ }^{2}$ Key Lab of Visual Damage and Regeneration \& Restoration, Chongqing 400038, China.

Received: 25 July 2021 Accepted: 10 November 2021

Published online: 11 February 2022

\section{References}

1. Narayan DS, Wood JPM, Chidlow G, Casson RJ. A review of the mechanisms of cone degeneration in retinitis pigmentosa. Acta Ophthalmol. 2016:94(8):748-54.

2. Zhang Q. Retinitis pigmentosa. Asia-Pac J Ophthalmol. 2016;5(4):265-71.

3. Garoon RB, Stout JT. Update on ocular gene therapy and advances in treatment of inherited retinal diseases and exudative macular degeneration. Curr Opin Ophthalmol. 2016;27(3):268-73.

4. Vaidya A, Borgonovi E, Taylor RS, Sahel JA, Rizzo S, Stanga P, et al. The cost-effectiveness of the Argus II retinal prosthesis in retinitis Pigmentosa patients. BMC Ophthalmol. 2014;14:49.

5. He Y, Zhang Y, Su G. Recent advances in treatment of retinitis pigmentosa. Curr Stem Cell Res Ther. 2015;10(3):258-65.

6. Gao J, Hussain RM, Weng CY. Voretigene neparvovec in retinal diseases: a review of the current clinical evidence. Clin Ophthalmol. 2020;14:3855-69.

7. Maguire AM, Bennett J, Aleman EM, Leroy BP, Aleman TS. Clinical perspective: treating RPE65- associated retinal dystrophy. Mol Ther. 2020:29(2):442-63.

8. Kang C, Scott LJ. Voretigene neparvovec: a review in RPE65 mutationassociated inherited retinal dystrophy. Mol Diagn Ther. 2020;24(4):487-95.

9. Mo M, Wang S, Zhou Y, Li H, Wu Y. Mesenchymal stem cell subpopulations: phenotype, property and therapeutic potential. Cell Mol Life Sci. 2016;73(17):3311-21.

10. Markov A, Thangavelu L, Aravindhan S, Zekiy AO, Jarahian M, Chartrand MS, et al. Mesenchymal stem/stromal cells as a valuable source for the treatment of immune-mediated disorders. Stem Cell Res Ther. 2021;12(1):192.

11. Hong HS, Kim YH, Son Y. Perspectives on mesenchymal stem cells: tissue repair, immune modulation, and tumor homing. Arch Pharm Res. 2012:35(2):201-11.

12. Liu DN, Yin ZQ, Wu N, Wang YH, Chen LF. Rat bone marrow stromal cells express retinal phenotypic markers following different induction protocols. Ophthalmic Res. 2009:41(4):186-93.

13. Duan $P, X u H$, Zeng $Y$, Wang $Y, Y$ in $Z Q$. Human bone marrow stromal cells can differentiate to a retinal pigment epithelial phenotype when cocultured with pig retinal pigment epithelium using a transwell system. Cell Physiol Biochem. 2013;31(4-5):601-13.

14. Ng TK. Progress of mesenchymal stem cell therapy for neural and retinal diseases. World J Stem Cells. 2014;6(2):111.

15. Gu X, Yu X, Zhao C, Duan P, Zhao T, Liu Y, et al. Efficacy and safety of autologous bone marrow mesenchymal stem cell transplantation in patients with diabetic retinopathy. Cell Physiol Biochem. 2018;49(1):40-52.

16. Moll G, Ankrum JA, Kamhieh-Milz J, Bieback K, Ringdén O, Volk H-D, et al. Intravascular mesenchymal stromal/stem cell therapy product diversification: time for new clinical guidelines. Trends Mol Med. 2019;25(2):149-63.

17. Ra JC, Shin IS, Kim SH, Kang SK, Kang BC, Lee HY, et al. Safety of intravenous infusion of human adipose tissue-derived mesenchymal stem cells in animals and humans. Stem Cells Dev. 2011;20(8):1297-308.

18. Tzameret A, Sher I, Belkin M, Treves AJ, Meir A, Nagler A, et al. Epiretinal transplantation of human bone marrow mesenchymal stem cells rescues 
retinal and vision function in a rat model of retinal degeneration. Stem Cell Res. 2015;15(2):387-94.

19. Jian Q, Li Y, Yin ZQ. Rat BMSCs initiate retinal endogenous repair through NGF/TrkA signaling. Exp Eye Res. 2015;132:34-47.

20. Satarian L, Nourinia R, Safi S, Kanavi MR, Baharvand H. Intravitreal injection of bone marrow mesenchymal stem cells in patients with advanced retinitis pigmentosa; a safety study. J Ophthalmic Vis Res. 2017;12(1):58.

21. Zhao T, Liang Q, Meng X, Duan P, Wang F, Li S, et al. Intravenous infusion of umbilical cord mesenchymal stem cells maintains and partially improves visual function in patients with advanced retinitis pigmentosa. Stem Cells Dev. 2020;29(16):1029-37.

22. Li T, Xia M, Gao Y, Chen Y, Xu Y. Human umbilical cord mesenchymal stem cells: an overview of their potential in cell-based therapy. Expert Opin Biol Ther. 2015;15(9):1293-306.

23. de Witte SFH, Merino AM, Franquesa M, Strini T, van Zoggel JAA, Korevaar SS, et al. Cytokine treatment optimises the immunotherapeutic effects of umbilical cord-derived MSC for treatment of inflammatory liver disease. Stem Cell Res Ther. 2017;8(1):140.

24. Matheakakis A, Batsali A, Papadaki HA, Pontikoglou CG. Therapeutic implications of mesenchymal stromal cells and their extracellular vesicles in autoimmune diseases: from biology to clinical applications. Int J Mol Sci. 2021;22(18):10132.

25. de Witte SFH, Luk F, Sierra Parraga JM, Gargesha M, Merino A, Korevaar SS, et al. Immunomodulation by therapeutic mesenchymal stromal cells (MSC) is triggered through phagocytosis of MSC by Monocytic cells. Stem Cells. 2018;36(4):602-15.

26. Muller L, Tunger A, Wobus $\mathrm{M}$, von Bonin $\mathrm{M}$, Towers $\mathrm{R}$, Bornhauser $\mathrm{M}$, et al. Immunomodulatory properties of Mesenchymal stromal cells: an update. Front Cell Dev Biol. 2021;9:637725.

27. Podesta MA, Remuzzi G, Casiraghi F. Mesenchymal stromal cells for transplant tolerance. Front Immunol. 2019;10:1287.

28. Wang LT, Liu KJ, Sytwu HK, Yen ML, Yen BL. Advances in mesenchymal stem cell therapy for immune and inflammatory diseases: use of cell-free products and human pluripotent stem cell-derived mesenchymal stem cells. Stem Cells Transl Med. 2021;10(9):1288-303.

29. Eggenhofer E, Benseler V, Kroemer A, Popp FC, Geissler EK, Schlitt HJ, et al. Mesenchymal stem cells are short-lived and do not migrate beyond the lungs after intravenous infusion. Front Immunol. 2012;3:297.

30. Saat TC, van den Engel S, Bijman-Lachger W, Korevaar SS, Hoogduijn MJ, ljzermans JNM, et al. Fate and effect of intravenously infused mesenchymal stem cells in a mouse model of hepatic ischemia reperfusion injury and resection. Stem Cells Int. 2016;2016:5761487.

31. Strauss O, Stumpff F, Mergler S, Wienrich M, Wiederholt M. The Royal College of surgeons rat: an animal model for inherited retinal degeneration with a still unknown genetic defect. Acta Anat (Basel). 1998;162(2-3):101-11.

32. Gal A, Li Y, Thompson DA, Weir J, Orth U, Jacobson SG, et al. Mutations in MERTK, the human orthologue of the RCS rat retinal dystrophy gene, cause retinitis pigmentosa. Nat Genet. 2000;26(3):270-1.

33. Dominici M, Le Blanc K, Mueller I, Slaper-Cortenbach I, Marini FC, Krause DS, et al. Minimal criteria for defining multipotent mesenchymal stromal cells. The International Society for Cellular Therapy position statement. Cytotherapy. 2006;8(4):315-7.

34. Chao K, Zhang S, Qiu Y, Chen X, Zhang X, Cai C, et al. Human umbilical cord-derived mesenchymal stem cells protect against experimental colitis via CD5+ B regulatory cells. Stem Cell Res Ther. 2016;7(1):109.

35. Cortes-Dericks L. Human mesenchymal stem cell-derived conditioned medium: perspectives for therapeutic application in lung cancers. J Tissue Sci Eng. 2017;8(2):25.

36. Nagamura-Inoue T. Umbilical cord-derived mesenchymal stem cells: their advantages and potential clinical utility. World J Stem Cells. 2014;6(2):195.

37. El Omar R, Beroud J, Stoltz JF, Menu P, Velot E, Decot V. Umbilical cord mesenchymal stem cells: the new gold standard for mesenchymal stem cell-based therapies? Tissue Eng B Rev. 2014;20(5):523-44.

38. Li X, Bai J, Ji X, Li R, Xuan Y, Wang Y. Comprehensive characterization of four different populations of human mesenchymal stem cells as regards their immune properties, proliferation and differentiation. Int J Mol Med. 2014;34(3):695-704.

39. Borys-Wojcik S, Brazert M, Jankowski M, Ozegowska K, Chermula B, Piotrowska-Kempisty $\mathrm{H}$, et al. Human Wharton's jelly mesenchymal stem cells: properties, isolation and clinical applications. J Biol Regul Homeost Agents. 2019;33(1):119-23.
40. Batsali AK, Kastrinaki MC, A. Papadaki H, Pontikoglou C. Mesenchymal stem cells derived from Wharton's jelly of the umbilical cord: biological properties and emerging clinical applications. Curr Stem Cell Res Ther. 2013;8(2):144-55.

41. Meng F, Xu R, Wang S, Xu Z, Zhang C, Li Y, et al. Human umbilical cordderived mesenchymal stem cell therapy in patients with COVID-19: a phase 1 clinical trial. Signal Transduct Target Ther. 2020;5(1):172.

42. Wang L, Huang S, Li S, Li M, Shi J, Bai W, et al. Efficacy and safety of umbilical cord Mesenchymal stem cell therapy for rheumatoid arthritis patients: A prospective phase I/II study. Drug Des Devel Ther. 2019;13:4331-40.

43. Lee RH, Pulin AA, Seo MJ, Kota DJ, Ylostalo J, Larson BL, et al. Intravenous hMSCs improve myocardial infarction in mice because cells embolized in lung are activated to secrete the anti-inflammatory protein TSG-6. Cell Stem Cell. 2009;5(1):54-63.

44. Fischer UM, Harting MT, Jimenez F, Monzon-Posadas WO, Xue H, Savitz SI, et al. Pulmonary passage is a major obstacle for intravenous stem cell delivery: the pulmonary first-pass effect. Stem Cells Dev. 2009;18(5):683-92.

45. Kim M, Bae YK, Um S, Kwon JH, Kim GH, Choi SJ, et al. A small-sized population of human umbilical cord blood-derived mesenchymal stem cells shows high stemness properties and therapeutic benefit. Stem Cells Int. 2020;2020:1-17.

46. Chin SP, Mohd-Shahrizal MY, Liyana MZ, Then KY, Cheong SK. High dose of intravenous allogeneic umbilical cord-derived Mesenchymal stem cells (CLV-100) infusion displays better Immunomodulatory effect among healthy volunteers: A phase 1 clinical study. Stem Cells Int. 2020;2020:8877003.

47. Stefanska K, Ozegowska K, Hutchings G, Popis M, Moncrieff L, Dompe C, et al. Human Wharton's jelly-cellular specificity, Stemness potency, animal models, and current application in human clinical trials. J Clin Med. 2020;9(4):1102.

48. Nystedt J, Anderson $\mathrm{H}$, Tikkanen J, Pietila M, Hirvonen T, Takalo R, et al. Cell surface structures influence lung clearance rate of systemically infused mesenchymal stromal cells. Stem Cells. 2013;31(2):317-26.

49. Zanetti A, Grata M, Etling EB, Panday R, Villanueva FS, Toma C. Suspension-expansion of bone marrow results in small mesenchymal stem cells exhibiting increased transpulmonary passage following intravenous administration. Tissue Eng C Methods. 2015;21(7):683-92.

50. Ge J, Guo L, Wang S, Zhang Y, Cai T, Zhao RCH, et al. The size of mesenchymal stem cells is a significant cause of vascular obstructions and stroke. Stem Cell Rev Rep. 2014;10(2):295-303.

51. Wang L, Li P, Tian Y, Li Z, Lian C, Ou Q, et al. Human umbilical cord mesenchymal stem cells: subpopulations and their difference in cell biology and effects on retinal degeneration in RCS rats. Cell Physiol Biochem. 2018;17(6):421-35.

52. Qu L, Gao L, Xu H, Duan P, Zeng Y, Liu Y, et al. Combined transplantation of human mesenchymal stem cells and human retinal progenitor cells into the subretinal space of RCS rats. Sci Rep. 2017;7(1):199.

53. Jin SZ, Liu BR, Xu J, Gao FL, Hu ZJ, Wang XH, et al. Ex vivo-expanded bone marrow stem cells home to the liver and ameliorate functional recovery in a mouse model of acute hepatic injury. Hepatobil Pancreat Dis Int. 2012;11(1):66-73.

54. Kang SK, Shin IS, Ko MS, Jo JY, Ra JC. Journey of mesenchymal stem cells for homing: strategies to enhance efficacy and safety of stem cell therapy. Stem Cells Int. 2012;2012:342968.

55. Wang S, Lu B, Girman S, Duan J, McFarland T, Zhang QS, et al. Non-invasive stem cell therapy in a rat model for retinal degeneration and vascular pathology. PLoS One. 2010;5(2):e9200.

56. Gnecchi M, Danieli P, Malpasso G, Ciuffreda MC. Paracrine mechanisms of mesenchymal stem cells in tissue repair. Methods Mol Biol. 2016;1416:123-46.

57. Jung H, Chun MS, Chang MS. Sorting of human mesenchymal stem cells by applying optimally designed microfluidic chip filtration. Analyst. 2015;140(4):1265-74.

58. Majore I, Moretti P, Hass R, Kasper C. Identification of subpopulations in mesenchymal stem cell-like cultures from human umbilical cord. Cell Commun Signal. 2009;7:6.

\section{Publisher's Note}

Springer Nature remains neutral with regard to jurisdictional claims in published maps and institutional affiliations. 